\title{
A Suspected Case of Silodosin-Induced Erythroderma
}

\author{
Annie Liu • Lyne Giroux
}

Published online: 4 February 2015

(C) The Author(s) 2015. This article is published with open access at Springerlink.com

\begin{abstract}
An 86-year-old man developed a suspected severe erythroderma during treatment with silodosin (dosage unknown) for benign prostatic enlargement. Two weeks after starting silodosin, he developed a total-body scaling dermatitis. A biopsy was planned but the patient improved at his subsequent visit and it was not taken. Silodosin was discontinued and the patient received UVB phototherapy, clobetasol ointment, and several bland and protective skin-care measures. One week after the initial presentation, the patient demonstrated improvement in his total-body scaling. Based on these findings, the patient was diagnosed with a suspected silodosin-induced erythroderma. Due to limitations in the patient's clinical history and investigations, a Naranjo assessment score was not obtainable.
\end{abstract}

A. Liu

Faculty of Medicine, The University of Toronto,

Toronto, ON, Canada

A. Liu $(\bowtie)$

King's College Circle, Medical Sciences Building,

Toronto, ON, Canada

e-mail: msa.liu@mail.utoronto.ca

L. Giroux

Department of Dermatology, The Northern Ontario School of

Medicine, Sudbury, ON, Canada

\section{Key Points}

Erythroderma is a complex condition of skin inflammation that causes total-body scaling and erythema.

Prescribers should advise patients taking silodosin to watch for a total-body, coalescing, scaling, and inflammatory rash.

The development of a total-body, coalescing, scaling, and inflammatory rash requires immediate discontinuation and avoidance of silodosin, which usually allows for a complete recovery.

\section{Background}

Silodosin is an $\alpha_{1}$-adrenoceptor antagonist with high uroselectivity that is a relatively new medication used for the treatment of benign prostatic enlargement (BPE) [1]. Current commonly reported adverse effects include a decrease or absence of semen during intercourse, dizziness, diarrhea, postural hypotension, headache, swelling of the throat and nasal passages, and stuffy nose [2]. BPE is one of the most common conditions associated with aging in men, affecting as many as $70 \%$ of men aged $61-70$ years and $90 \%$ of men aged $81-90$ years [3]. The presence of any dangerous or complicating adverse effects could therefore be harmful to a proportion of the elderly population. We report a case of a suspected severe silodosininduced erythrodermic drug rash. In completing a literature search through PubMed and MEDLINE using the keywords "silodosin" and "erythroderma", we found no prior 
report of this adverse drug reaction. To the best of our knowledge, this is the first time it has been described in the literature.

\section{Case Presentation}

An 86-year-old man presented with a 5-month history of a severe dermatitis affecting the entire body. The patient was not in acute distress, but appeared unwell and exhibited chills. There was no lymphadenopathy present. He denied any other systemic symptoms. A symmetrical, diffuse erythema was present with overlying scale covering $>90 \%$ of the body surface area. His skin was warm to the touch. There was no mucosal membrane involvement. The rash began in the groin and had since spread to the rest of the body. It was previously treated with several over-thecounter creams with no amelioration of symptoms. Silodosin had been started for prostatic enlargement 2 weeks before the rash began. In addition to silodosin, the patient was taking ramipril, cyclobenzaprine, atorvastatin, rabeprazole, levothyroxine, meloxicam, hydrocortisone valerate, an antihistamine of unknown name, ipratropium, and bacitracin/polymyxin (exact formulation of Polysporin $^{\circledR}$ unknown). The patient was unable to provide the dosage of any of his medications. With the exception of silodosin, the patient did not report having recently started any of his other medications. Past medical, dermatologic, and family history was unremarkable. The patient quit smoking approximately 2 months prior. Figures 1 and 2 are photographs of the patient's initial presentation.

\section{Investigations}

A biopsy was scheduled to be performed in the week following the patient's initial visit, but the patient improved with treatment so it was not performed. No other laboratory tests were sent off.

\section{Differential Diagnosis}

The differential diagnosis included drug-induced erythroderma, allergic contact dermatitis, psoriasis, Sézary syndrome, or erythrodermic cutaneous $\mathrm{T}$ cell lymphoma. The latter four are common dermatoses that can eventually progress to erythroderma. Like an erythrodermic drug reaction, allergic contact dermatitis can also present as confluent scales and plaques of erythema, edema, and eventual lichenification shortly after exposure to an offending agent [4]. Similarly, acute guttate psoriasis can exhibit scales and scattered rash-like lesions that tend to coalesce [4]. In

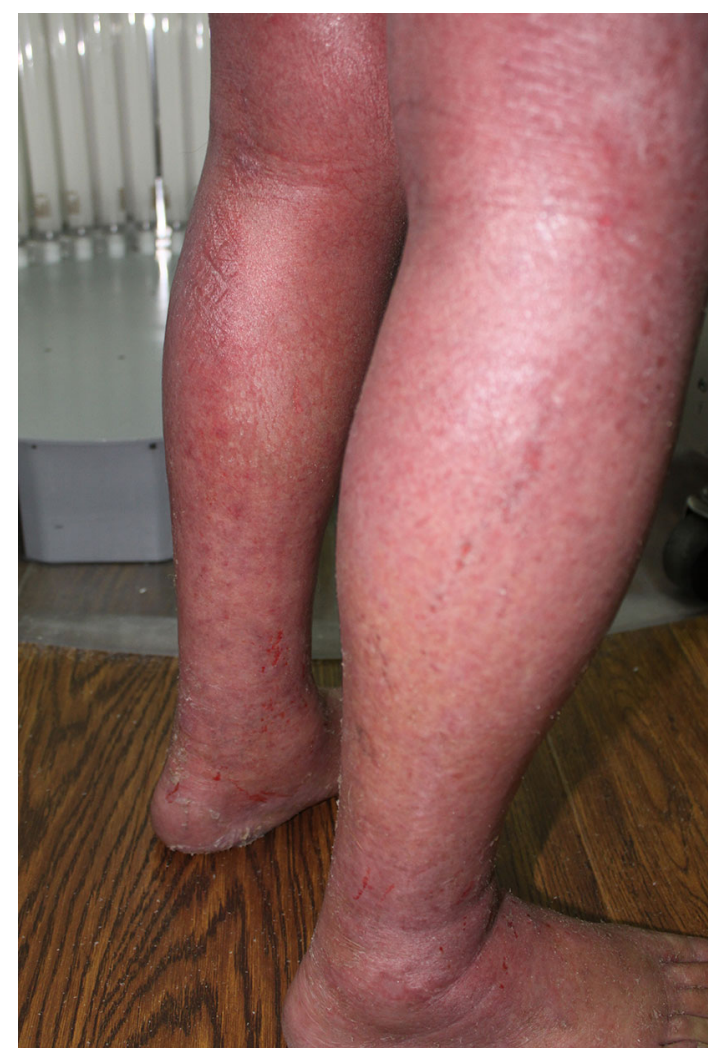

Fig. 1 Initial patient presentation

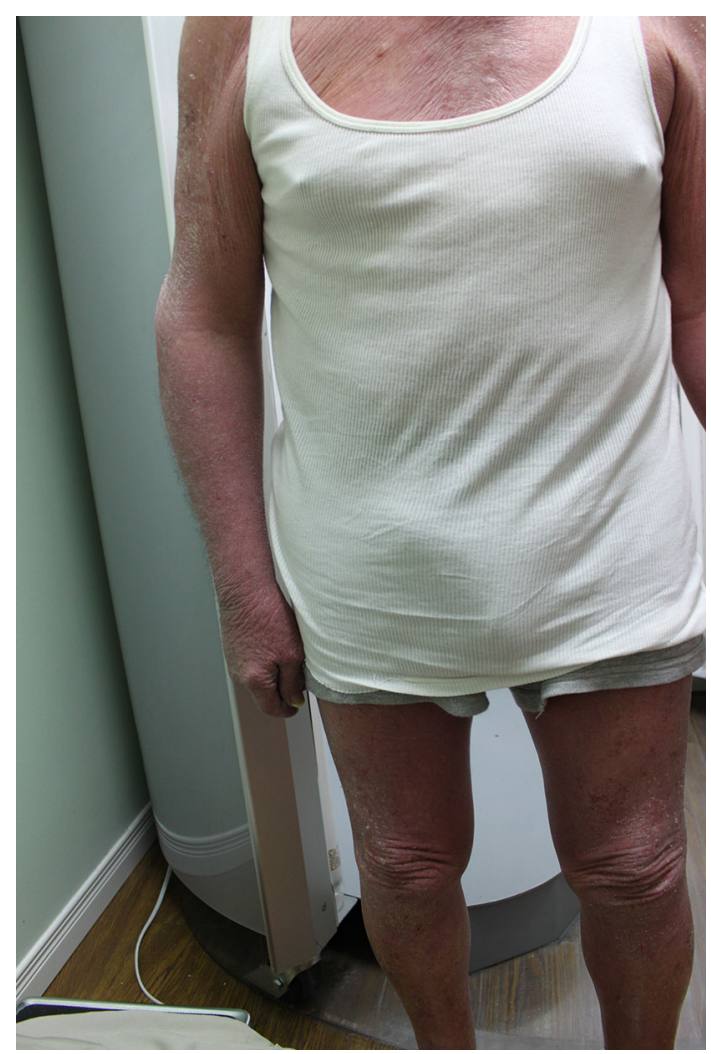

Fig. 2 Initial patient presentation 
addition, the characteristic shivering, total-body erythema, and generalized scaling and lichenification of the skin are also seen in Sézary syndrome [4]. Finally, like erythroderma, early cutaneous $\mathrm{T}$ cell lymphoma can also present with illdefined, scaling patches of erythema [4].

\section{Treatment}

Treatment options were discussed and explained to the patient. Treatment began with an immediate (starting the day of his presentation) withdrawal of silodosin for 6 weeks. UVB phototherapy was then provided to accelerate skin healing. Lasix $^{\circledR} 40 \mathrm{mg} /$ day for 1 week as well as $18-20 \mathrm{mmHg}$ compression stockings were given to treat the edema.

The patient was also provided with medications and guidelines to ease the discomfort of the rash. This included clobetasol ointment three times daily after a short shower for 4 weeks for reduction of inflammation and itch, bleach baths three times per week to prevent secondary impetiginization with Staphylococcus aureus $\left(1 / 2\right.$ cup of $\mathrm{Javex}^{\circledR}$ in a full lukewarm bath for 10 min followed by a rinse, a pat dry, application of a moisturizer, and clobetasol); the use of $\mathrm{CeraVe}^{\circledR}$ or Cliniderm ${ }^{\circledR}$ cleanser and moisturizer for skin barrier protection and symptomatic relief; hydroxyzine hydrochloride $25 \mathrm{mg}$ orally in the evening for trouble sleeping; and cessation of all other creams and shampoos to lessen the chance of exposure to potential contact allergies.

Based on the patient's improvement of his total-body erythroderma within 1 week of implementing the aforementioned treatments, we consider silodosin withdrawal, topical clobetasol ointment, and UVB treatment an effective approach to silodosin-induced erythrodermic rash.

\section{Outcome and Follow-Up}

One week after the initial presentation, silodosin withdrawal, and treatment (outlined above), the patient showed great signs of improvement of his total-body erythroderma. Figure 3 is a photograph of the patient's outcome after 1 week.

\section{Discussion}

Although a biopsy was not performed for confirmation, silodosin-induced erythroderma is the most likely diagnosis, as none of the patient's other medications nor the overthe-counter products could account for his full-body erythroderma. Given that, with the exception of silodosin, the patient had not recently started any other medication, it was most likely that silodosin was the main cause of his

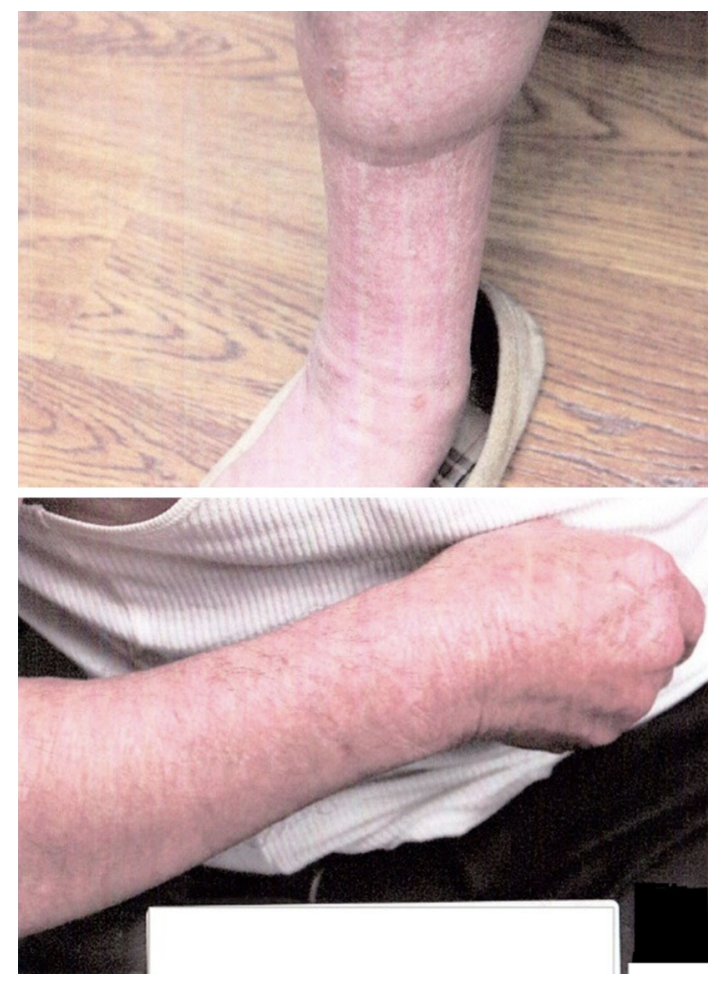

Fig. 3 One week post-treatment

erythroderma. In fact, silodosin had been started 2 weeks prior to the onset of the patient's erythroderma. Furthermore, when silodosin was withdrawn and a number of other symptomatic treatments applied, the erythroderma demonstrated rapid and significant improvement. Nevertheless, several other causes for the patient's presentation and resolution of symptoms remain possible. Firstly, it is possible that silodosin created a drug-drug interaction with another medication that the patient was already taking. Specifically, atorvastatin can increase the level or effect of silodosin by the P-glycoprotein [multidrug resistance gene (MDR1)] efflux transporter [5]. Additionally, it is possible that the patient developed an idiopathic erythroderma that coincided with the starting of silodosin. Although erythroderma can be idiopathic [4], the temporal link between the patient's symptoms and the starting and cessation of silodosin decrease the likeliness of this explanation. Finally, it is possible that one or more of the UVB phototherapy or symptomatic treatments (clobetasol ointment, belch baths, cleanser and moisturizer, hydroxyzine hydrochloride) contributed to or caused the patient's recovery, as these treatments work as anti-inflammatory, bland, and protective skin-care measures.

Erythroderma is a condition of inflammation and skin dysmetabolism that causes total-body scaling and erythema [6]. It carries a significant risk of morbidity and mortality even when properly managed, primarily because of its metabolic burden and complications [6]. An increasing 
incidence of erythroderma may be due to our era's rising production of new drugs. Additional drugs linked to erythroderma include anticonvulsants, antihypertensives, antibacterials, and calcium channel antagonists [7].

Currently, the pathophysiology of erythroderma is incompletely understood. It is suggested that an increase in expression of adhesion molecules (e.g., vascular cell adhesion protein-1) and their ligands may stimulate inflammation and increased epidermal proliferation $[6,8]$. As well, the interaction of cytokines and adhesion molecules increases the epidermal turnover rate and leads to additional loss of epidermal cells at the surface $[6,9]$. In our patient, these underlying mechanisms may explain his presentation, as the exfoliating nature of his rash indicated increased inflammation as well as epidermal proliferation and loss at the skin surface. Additionally, our patient's malaise and diffuse chills can be attributed to an increased perfusion in his skin, which caused heat loss, hypothermia, and a compensatory rise in basal metabolic rate [10].

Erythroderma typically presents as erythematous patches, which eventually coalesce to produce extensive totalbody erythema [9]. The skin is bright red, scaly, dry, and warm to the touch, but may also appear thin and glossy [6]. Thickening of the skin due to edema and lichenification may cause an appearance of skin tightness [6].

The initial management of erythroderma, regardless of etiology, should include bland and protective skin-care measures [11, 12], and mid-potency corticosteroids, preferably ointments. For a drug-induced erythrodermic rash, the most important aspect of treatment is to immediately discontinue and avoid all precipitants and irritants. Edema can be treated with diuretics and secondary infections should be treated with antibacterials. Because erythroderma may continue to progress until the underlying cause is treated, early identification of the etiology is essential to successful management. In severe cases where there is notable hemodynamic instability, which was not present in our current case, the patient should also receive replacement of fluid, electrolyte, and nutrition losses. Although this is the first case of a suspected silodosin-induced erythroderma with recovery after drug withdrawal, druginduced erythrodermic patients usually recover completely with prompt diagnosis and treatment [11].

Erythroderma is a complex condition for which the prognosis depends on the etiology. Drug-induced erythrodermas usually recover completely with prompt diagnosis and removal of the offending agent [11]. Although erythroderma has been attributed to countless drug reactions (e.g., anticonvulsants, antihypertensives), it has never been described for silodosin. Patients should exercise caution with its use, particularly when used simultaneously with atorvastatin, and watch for signs of a total-body, coalescing, scaling, and inflammatory rash. The development of such symptoms requires immediate discontinuation and avoidance of the offending agent.

Acknowledgments Annie Liu and Dr. Lyne Giroux have no acknowledgements to make. Annie Liu and Dr. Lyne Giroux declare that they have no conflicts of interest. No financial support was received for the conduct of this study or preparation of this manuscript.

Consent Written informed consent was obtained from the patient for publication of this case report and accompanying images. A copy of the written consent is available for review by the Editor-in-Chief of this journal.

Open Access This article is distributed under the terms of the Creative Commons Attribution Noncommercial License which permits any noncommercial use, distribution, and reproduction in any medium, provided the original author(s) and the source are credited.

\section{References}

1. Watson Pharmaceuticals, Inc.: Watson receives US FDA approval for RAPAFLO(TM) (silodosin) for the treatment of benign prostatic hyperplasia (BPH). http://ir.actavis.com/phoenix. zhtml $\mathrm{c}=65778 \& \mathrm{p}=$ irol-newsArticle $\& \mathrm{ID}=1207177$. Accessed 10 Aug 2013.

2. Watson Pharmaceuticals, Inc.: Important safety information. 2013. https://www.rapaflo.com/hcp-role-primary.asp. Accessed 10 Aug 2013.

3. McVary, KT. BPH: epidemiology and comorbidities. Am J Manag Care. 2006;12(5 Suppl):S122-8.

4. Wolff K, Johnson RA. Fitzpatrick's color atlas and synopsis of clinical dermatology. 6th ed. New York: The McGraw Hill Companies, Inc; 2009.

5. Medscape: Silodosin (Rx)—Rapaflo. Drug interactions. http:// reference.medscape.com/drug/rapaflo-silodosin-999100\#3. Accessed 7 Oct 2014.

6. Okoduwa C, Lambert WC, Schwartz RA, et al. Erythroderma: review of a potentially life-threatening dermatosis. Indian $\mathrm{J}$ Dermatol. 2009;54(1):1-6.

7. Rubins AY, Hartmane IV, Lielbriedis YM, et al. Therapeutic options for erythroderma. Cutis. 1992;49(6):424-6.

8. Sigurdsson V, de Vries IJ, Toonstra $\mathrm{J}$, et al. Expression of VCAM-1, ICAM-1, E-selectin, and P-selectin on endothelium in situ in patients with erythroderma, mycosis fungoides and atopic dermatitis. J Cutan Pathol. 2000;27(9):436-40.

9. Sehgal VN, Srivastava G, Sardana K. Review erythroderma/exfoliative dermatitis: a synopsis. Int J Dermatol. 2004;43(1): $39-47$.

10. Umar SH, Kelly AP. Erythroderma (generalized exfoliative dermatitis). Medscape. 2012. http://emedicine.medscape.com/ article/1106906-overview\#a0104. Accessed 22 Sep 2013.

11. Rothe MJ, Bialy TL, Grant-Kels JM. Erythroderma. Dermatol Clin. 2000;18(3):405-15.

12. Rothe MJ, Bernstein ML, Grant-Kels JM. Life-threatening erythroderma: diagnosing and treating the "red man". Clin Dermatol. 2005;23:206-17. 